\title{
Extensive Sinonasal Rhabdomyosarcoma in an Adult
}

\author{
Muhammad Adam Shariff ${ }^{1}$, Jeyasakthy Saniasiaya ${ }^{2}$
}

\begin{abstract}
Aim and objective: This study aimed to highlight the importance of diagnosing sinonasal rhabdomyosarcoma (RMS) in an adult patient.

Background: Rhabdomyosarcoma is a malignant tumor that originates from striated muscle. It is known to be the most common sarcoma in children. Occurrence among adults especially above 50 years of age is rare.

Case description: Herein, we report on an adult patient who presented with a 1-month history of unilateral nasal obstruction and epiphora which turned out to be extensive sinonasal RMS extending intracranially.

Conclusion: The patient was treated referred to the oncology unit for chemotherapy.

Clinical significance: We would like to highlight that following the aggressive nature of sinonasal RMS, prompt management is undertaken to improve the survival rate.

Keywords: Alveolar rhabdomyosarcoma, Chemotherapy, Sinonasal carcinoma.

Clinical Rhinology An International Journal (2020): 10.5005/jp-journals-10013-1368
\end{abstract}

\section{BACKGROUND}

Rhabdomyosarcoma (RMS) is the most common soft tissue malignancy in children and is considered extremely rare among adults. It is known to arise from myogenic cells. In the head and neck, parameningeal RMS is noted to have a worse prognosis compared with non-parameningeal subsites and tends skull base infiltration and intracranial involvement. Imaging study and histopathological examination is the fundamental step to diagnose RMS.

\section{Case Description}

A previously healthy 53-year-old woman presented with a 1-month history of left-sided nasal obstruction and epiphora. In addition to that, the patient is noted to have a significant weight loss of $15 \mathrm{~kg}$ within the past 2 months. Besides that, the patient has no epistaxis, no diplopia, or any signs of increased intracranial pressure. The patient also has no ear or throat symptoms. There is no family history of malignancy.

On examination, the patient appears comfortable, not cachexiclooking. Left eye proptosis was noted with normal extraocular eye movement. There appears to be no obvious external nose deformity. Nasoendoscopy revealed a fungating mass occupying the entire left nasal cavity which bleeds on touch. The right nasal cavity revealed no nasal mass. Intraoral examination was unremarkable. Neck examination along with other systemic and cranial nerve examinations was intact. Biopsy of the left nasal mass was done under local anesthesia which revealed tissue lined by ciliated stratified columnar epithelium with nests of malignant cells displaying pleomorphic nucleoli, surrounding stroma appears edematous and inflammatory infiltrates mainly eosinophils, neutrophils, and lymphoplasmacytic cells. Further immunohistochemistry study revealed malignant cells that are diffusely positive for vimentin, myogenin, and myoD1 while focally positive for desmin (Fig. 1). Further staining for $C K A E 1 / 3$, smooth muscle actin, s-100 protein, Melan A, HMB45, CD34, and factor VIII was found to be negative. The malignant cells were noted to be arranged in an alveolar pattern separated by fibrous septa which were suggestive of the alveolar type of sinonasal RMS.
1Department of Otorhinolaryngology, Hospital Selayang, Lebuhraya
Selayang-Kepong, Selayang, Selangor, Malaysia
${ }^{2}$ Department of Otorhinolaryngology, Faculty of Medicine, University
of Malaya, Jalan University, Kuala Lumpur, Malaysia
Corresponding Author: Jeyasakthy Saniasiaya, Department of
Otorhinolaryngology, Faculty of Medicine, University of Malaya, Jalan
University, Kuala Lumpur, Malaysia, Phone: +60126841123, e-mail:
drjeyasakthy83@gmail.com
How to cite this article: Shariff MA, Saniasiaya J. Extensive Sinonasal
Rhabdomyosarcoma in an Adult. Clin Rhinol An Int J 2020;13(1):
$21-23$.
Source of support: Nil
Conflict of interest: None

Computed tomography (CT) brain till abdomen demonstrated enhancing mass with cystic component arising from the left sinonasal region extending to the left frontal lobe and left orbit with an erosion of the adjacent bones. The lesion also appears to extend into the anterior cranial fossa and the left cerebral hemisphere through a breach at the left cribriform plate. Hence, a diagnosis of the alveolar type of RMS with intracranial extension was made.

The patient was subsequently referred to an oncology center and was started on chemotherapy.

\section{Discussion}

Rhabdomyosarcoma is a rare malignant tumor arising from a myogenic cell and is the most common soft tissue sarcoma in the pediatric population. ' Approximately $40 \%$ of RMS arises within the head and neck. In general, this entity can be classified further into orbital, parameningeal, and non-orbital non-parameningeal forms according to their anatomical location. ${ }^{1}$ Parameningeal RMS arises in the middle ear cleft, paranasal sinuses, nasal cavity, pterygopalatine, and infratemporal fossa region. It is reported to carry the worst prognosis as it tends to erode the base of the skull and also to extend into the intracranial region as well as perineural invasion. Moreover, poor response to treatment has been noted among the patients. ${ }^{1-3}$ 

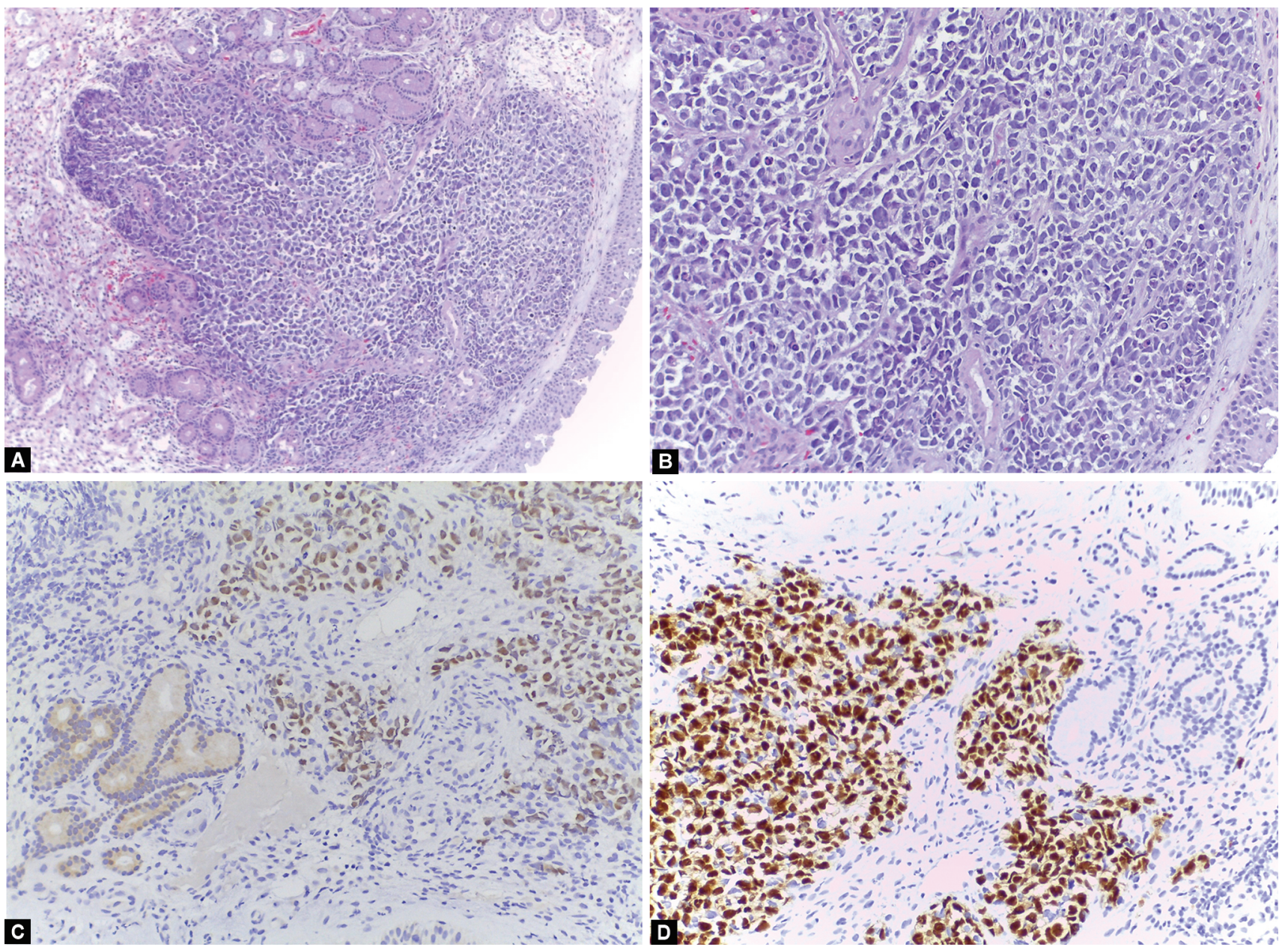

Figs 1A to D: (A) Presence of tumor cells in solid nests sparing the covering respiratory epithelium; (B) Cellular rounded blue cell tumor with subtle fibrous septa; (C) Myogenin stain is positive; (D) MyoD1 stain diffusely positive

Sinonasal RMS is a parameningeal type of RMS that often presents at an advanced stage. Oftentimes delayed presentation or diagnosis is following subtle signs and symptoms like epistaxis, rhinorrhea, nasal congestion as well as asymptomatic sinonasal mass. ${ }^{3}$ Our patient presented with unilateral nasal obstruction and epiphora which she noticed only for the past 1-month. Turner and Richmon ${ }^{4}$ published a retrospective review of head and neck RMS that included 248 patients with parameningeal tumors from the Surveillance, Epidemiology, and End Results (SEER) database which revealed 5 -year relative survival was $49.1 \%$. It is also noteworthy that the survival rate did not significantly change over the study period of 1973-2007. ${ }^{4}$

The gold standard in diagnosing this entity is based on histopathology and immunochemistry. Histologically, RMS can be differentiated based on its unique morphologic, genetic characteristics with important clinical and prognostic implications. Horn and Enterline in the 1950s classified RMS into alveolar, embryonal, botryoid, and pleomorphic. ${ }^{5}$ On the one hand, (Horn) embryonic type of RMS is the most common among pediatrics and carries the best prognosis. ${ }^{1,3,4}$ Alveolar type, on the other hand, carries the worst prognosis. Tumor cells appear small, round with large hyperchromatic nuclei and scanty pale cytoplasm surrounded by hyalinized stroma arranged in alveoli pattern. ${ }^{3,6,7}$ In immunohistochemistry, alveolar RMS shows strong and diffuse reaction with vimentin and muscle-specific antigens, such as, desmin, muscle actins (including smooth muscle isoforms), myogenin, and MyoD1. ${ }^{6,7}$ Immunohistochemistry in our patient demonstrated vimentin, myogenin, and myoD1 while focally positive for desmin.

Rhabdomyosarcoma warrants a multidisciplinary treatment approach that includes a combination of surgery, chemotherapy, and radiation. ${ }^{1,3}$ In parameningeal RMS, chemoradiotherapy is the treatment of choice with surgery having a limited role due to the relative inaccessibility of the lesions and associated surgical morbidity. ${ }^{1}$ In keeping with that, alveolar subtypes of RMS are known for its poor prognosis in view of aggressive presentation and increase the risk of metastasis and risk of intracranial extension. Having said that, surgical modality can be considered in localized, early stage disease, tumor debulking before chemoradiotherapy, and as a salvage treatment. Poor prognosis factor includes age, intracranial extension, the large size of the tumor, residual disease as well as metastasis. As a whole, the age of the patient as well as the alveolar subtype carries the worst prognosis.

\section{Conclusion}

Rhabdomyosarcoma albeit a rare head and neck tumor presents late following its subtle as well as aggressive presentation. Biopsy and imaging studies play an important role in providing valuable information as well as the mode of management. 


\section{ACKNOWLedgments}

We would like to acknowledge the whole team in the ORL department of Hospital Selayang.

\section{References}

1. Ilson S, Paulo V, Enrique P, et al. Sinonasal alveolar rhabdomyosarcoma in an adult patient: a case report and review of the literature. Cancer Ther Oncol Int J 2017;3(4):555619.

2. Hawkins DS, Anderson JR, Paidas CN, et al. Improved outcome for patients with middle ear rhabdomyosarcoma: a children's oncology group study. J Clin Oncol 2001;19(12):3073-3079. DOI: 10.1200/ JCO.2001.19.12.3073.
3. Thompson CF, Kim BJ, Lai C, et al. Sinonasal rhabdomyosarcoma: prognostic factors and treatment outcomes. Int Forum Allergy Rhinol 2013;3(8):678-683. DOI: 10.1002/alr.21157.

4. Turner JH, Richmon JD. Head and neck rhabdomyosarcoma: a critical analysis of population-based incidence survival data. Otolaryngol Head Neck Surg 2011;145(6):967-973. DOI: 10.1177/0194599811417063.

5. Horn RC, Enterline HT. Rhabdomyosarcoma: a clinicopathological study and classification of 39 cases. Cancer. 1958;11(1):181199. DOI: 10.1002/1097-0142(195801/02)11:1<181::AIDCNCR2820110130>3.0.CO;2-I.

6. Montone KT, Barr FG, Zhang PJ, et al. Embryonal and alveolar rhabdomyosarcoma of parameningeal sites in adults: a report of 13 cases. Int J Surg Pathol 2009;17(1):22-30. DOI: 10.1177/1066896908325876.

7. Covelloa R, Liccib S, Pichic FB, et al. Alveolar rhabdomyosarcoma of the paranasal cavity in an adult women. J Med Cases 2014;5(1):26-30. 